\title{
Submillimeter and millimeter masers
}

\author{
E. M. L. Humphreys \\ Harvard-Smithsonian CfA, 60 Garden Street, Cambridge, MA 02138, USA
}

\begin{abstract}
Despite theoretical predictions of the existence of many submillimeter masers, and some pioneering observational discoveries over the past few decades, these lines have remained relatively unstudied due to (i) challenges associated with observing at shorter wavelength; and, (ii) the lack of possibility of high $\left(<14^{\prime \prime}\right.$ at $\left.345 \mathrm{GHz}\right)$ angular resolution observations. With the advent of the SMA, the first submillimeter imaging array capable of sub-arcsecond resolution, APEX, and the promise of ALMA, opportunities are opening for performing new science with millimeter/submillmeter masers. In this talk, I will review recent work in the field - including extragalactic $\mathrm{H}_{2} \mathrm{O}$ millimeter masers, hydrogen recombination masers, submillimeter masers in star-forming regions, and in the envelopes of evolved stars - and discuss prospects for the future.
\end{abstract}

Keywords. masers, submillimeter

\section{Introduction}

Submillimeter masers exist in a wide range of astronomical environments, and provide the possibility to probe physical conditions, source dynamics and magnetic fields on small angular scales. They occur in several molecular and atomic species, including $\mathrm{H}_{2} \mathrm{O}, \mathrm{SiO}$, $\mathrm{H}$ (recombination), $\mathrm{CH}_{3} \mathrm{OH}, \mathrm{HCN}$, and $\mathrm{SiS}$, and can be very strong (e.g., $8000 \mathrm{Jy}$ for the $325 \mathrm{GHz} \mathrm{H}_{2} \mathrm{O}$ masers in W49N; Menten et al. 1990a). However, lack of angular resolution at submillimeter wavelengths has, until recently, been a serious obstacle to realizing the potential of the masers. Relating cm-wave maser emission observed on, say 0".001 scales, with that of the submillimeter maser emission on $>10^{\prime \prime}$ scales (at $345 \mathrm{GHz}$ ), has made it difficult to constrain and test the radiative transfer models that we will need to use in the Atacama Large Millimeter Array (ALMA) era to map out precise source temperature and density distributions.

The Submillimeter Array (SMA) on Mauna Kea, operating from 0.3 to $2 \mathrm{~mm}$, is the first instrument capable of imaging in the submillimeter on sub-arcsecond scales $(0$ "' 25 at $345 \mathrm{GHz})$, and ALMA will further transform maser science opportunities (see review by Wootten in these proceedings). In this review, I will discuss results for masers at wavelengths shorter than $1.6 \mathrm{~mm}(\nu>180 \mathrm{GHz})$, and future prospects for their observation using e.g., ALMA, the Herschel satellite, the Stratospheric Observatory for Infra-Red Astronomy (SOFIA) and submillimeter Very Long Baseline Interferometry (VLBI).

\section{2. (Sub)millimeter $\mathrm{H}_{2} \mathrm{O}$ masers}

The $\mathrm{H}_{2} \mathrm{O}$ masers detected to date, from rotational transitions within the vibrational ground state and within the $\nu_{2}=1$ bending mode, are listed in Table 1 and are marked on the energy level diagram in Figure 1. The most studied lines are those at 183, 321 and $325 \mathrm{GHz}$, despite the relatively low atmospheric transmission at 183 and $325 \mathrm{GHz}$ due to their low energies above ground state (Figure 2). These masers are believed to be collisionally-pumped by a subset of the conditions that pump $22 \mathrm{GHz}$ masers, for the 
Table 1. $\mathrm{H}_{2} \mathrm{O}$ masers

\begin{tabular}{|c|c|c|c|c|c|c|c|c|}
\hline $\begin{array}{l}\text { Freq. } \\
(\mathrm{GHz})\end{array}$ & $\begin{array}{c}\text { Transition } \\
\mathrm{J}_{k_{a}, k_{c}}-\mathbf{J}_{k_{a}, k_{c}}\end{array}$ & $\begin{array}{l}\text { Vib. } \\
\text { State }\end{array}$ & Species $^{1}$ & $\begin{array}{c}\mathrm{E}_{u} / \mathrm{k} \\
(\mathrm{K})\end{array}$ & $\mathrm{CSE}^{2}$ & $\mathrm{SFR}^{2}$ & $\mathrm{EXG}^{2}$ & Primary Reference \\
\hline 22.235 & $6_{16}-5_{23}$ & G & $\mathrm{O}$ & 644 & $\mathrm{Y}$ & $\mathrm{Y}$ & $\mathrm{Y}$ & Cheung et al. (1969) \\
\hline 96.261 & $4_{40}-5_{33}$ & $\nu_{2}=1$ & $\mathrm{P}$ & 3065 & $\mathrm{Y}$ & & & Menten \& Melnick (1989) \\
\hline 183.308 & $3_{13}-2_{20}$ & G & $\mathrm{P}$ & 205 & $\mathrm{Y}$ & $\mathrm{Y}$ & Y & Waters et al. (1980) \\
\hline 232.687 & $5_{50}-6_{43}$ & $\nu_{2}=1$ & $\mathrm{O}$ & 3463 & $\mathrm{Y}$ & & & Menten \& Melnick (1989) \\
\hline 293.439 & $6_{61}-7_{52}$ & $\nu_{2}=1$ & $\mathrm{O}$ & 3935 & $\mathrm{Y}$ & & & Menten et al. (2006) \\
\hline 321.226 & $10_{29}-9_{36}$ & $\mathrm{G}$ & $\mathrm{O}$ & 1862 & Y & Y & & Menten et al. (1990a) \\
\hline 325.153 & $5_{15}-4_{22}$ & G & $\mathrm{P}$ & 470 & $\mathrm{Y}$ & Y & & Menten et al. (1990b \\
\hline${ }^{3} 336.228$ & $5_{23}-6_{16}$ & $\nu_{2}=1$ & $\mathrm{O}$ & 2956 & $\mathrm{Y}$ & & & Feldman et al. (1993) \\
\hline 354.885 & $17_{412}-16_{710}$ & $\mathrm{G}$ & $\mathrm{O}$ & 5782 & $\mathrm{Y}$ & & & Feldman et al. (1991) \\
\hline 380.194 & $4_{14}-3_{21}$ & G & $\mathrm{O}$ & 324 & & Y & & Phillips et al. (1980) \\
\hline 437.347 & $7_{53}-6_{60}$ & G & $\mathrm{P}$ & 1525 & $\mathrm{Y}$ & & & Melnick et al. (1993) \\
\hline 439.151 & $6_{43}-5_{50}$ & G & $\mathrm{O}$ & 1089 & $\mathrm{Y}$ & Y & & Melnick et al. (1993) \\
\hline 470.889 & $6_{42}-5_{51}$ & G & $\mathrm{P}$ & 1091 & $\mathrm{Y}$ & $\mathrm{Y}$ & & Melnick et al. (1993) \\
\hline 658.007 & $1_{10}-1_{01}$ & $\nu_{2}=1$ & $\mathrm{O}$ & 2361 & $\mathrm{Y}$ & & & Menten \& Young (1995) \\
\hline
\end{tabular}

${ }^{1} \mathrm{O}=$ ortho- $\mathrm{H}_{2} \mathrm{O}$ (parallel hydrogen atom nuclear spins) and $\mathrm{P}=$ para- $\mathrm{H}_{2} \mathrm{O}$ (anti-parallel hydrogen nuclear spins). In thermal equilibrium, the two forms are present in an $\mathrm{O} / \mathrm{P}$ ratio of $3: 1$.

${ }^{2} \mathrm{CSE}=$ Circumstellar Envelope; $\mathrm{SFR}=$ Star Forming Region; $\mathrm{EXG}=$ Extragalactic

${ }^{3}$ Quasi-maser (Feldman et al. 1993), or thermal (Menten et al. 2006), emission toward VY CMa.

parameter space investigated by Neufeld \& Melnick (1991, hereafter NM91) and by Yates, Field \& Gray (1997, hereafter YFG97). However, Cernicharo et al. (1994, 1999, 2006a, 2006b) find that the 183 and $325 \mathrm{GHz}$ transitions can also be inverted in significantly lower temperature and density regimes of $\mathrm{T}_{k} \sim 40 \mathrm{~K}$ and $\mathrm{n}\left(\mathrm{H}_{2}\right)=10^{5}-10^{6} \mathrm{~cm}^{-3}$. NM91 and YFG97 are in broad agreement, except that YFG97 find that the masers at 439 and $470 \mathrm{GHz}$ are pumped by radiation from warm dust. Both NM91 and YFG97 make predictions for new $\mathrm{H}_{2} \mathrm{O}$ masers (e.g., at 448, 1296, \& $1322 \mathrm{GHz}$; also Deguchi 1977, Cooke \& Elitzur 1985, Deguchi \& Nguyen-Q-Rieu 1990, Humphreys et al. 2001), some of which could be observed using Herschel. NM91 and YFG97 do not include levels from the $\nu_{2}=1$ vibrationally-excited state, see Deguchi (1977), Deguchi \& Nguyen-Q-Rieu (1990) and Alcolea \& Menten (1993) for $\nu_{2}=1$ maser pumping models. Modelling of the $\nu_{2}=1$ masers is severely hampered by lack of relevant collisional excitation rates.

\section{1. $\mathrm{H}_{2} \mathrm{O}$ masers in evolved stars}

(Sub)millimeter masers at 183,321 and $325 \mathrm{GHz}$ are common in the circumstellar envelopes (CSEs) of evolved stars. $70 \%$ of the $22 \mathrm{GHz} \mathrm{H}_{2} \mathrm{O}$ maser sources observed by Yates, Cohen \& Hills (1995) also have $\mathrm{H}_{2} \mathrm{O}$ maser emission at 321 and $325 \mathrm{GHz}$. Singledish linewidths of 22 and $325 \mathrm{GHz}$ masers have similar extents and peak flux densities, whereas $321 \mathrm{GHz}$ maser line widths are narrower and weaker by a factor of a few (an exception is emission from R Aqr, a Mira variable in a symbiotic binary; Ivison et al. 1998). $321 \mathrm{GHz}$ emission likely originates from a subset of the conditions that give rise to the 22 and $325 \mathrm{GHz}$ emission, close to the central star. The $321 \mathrm{GHz}$ line is generally more variable than the 22 and $325 \mathrm{GHz}$ emission and variations in the 22, 321 and $325 \mathrm{GHz}$ masers are not particularly well-correlated (in some cases they are completely anti-correlated; Yates et al. 1996). For $183 \mathrm{GHz} \mathrm{H}_{2} \mathrm{O}$ masers, González-Alfonso et al. (1998) find that variability of the line profile and flux from one epoch to another is small in comparison with that of $22 \mathrm{GHz}$ masers in a study of 23 evolved stars. As for $22 \mathrm{GHz}$ $\mathrm{H}_{2} \mathrm{O}$ masers, in stars of low mass-loss rates $(\dot{M})$ the $183 \mathrm{GHz}$ emission peaks at a velocity similar to that of the star, whilst in stars with high $\dot{M}$ the emission peaks at velocities 


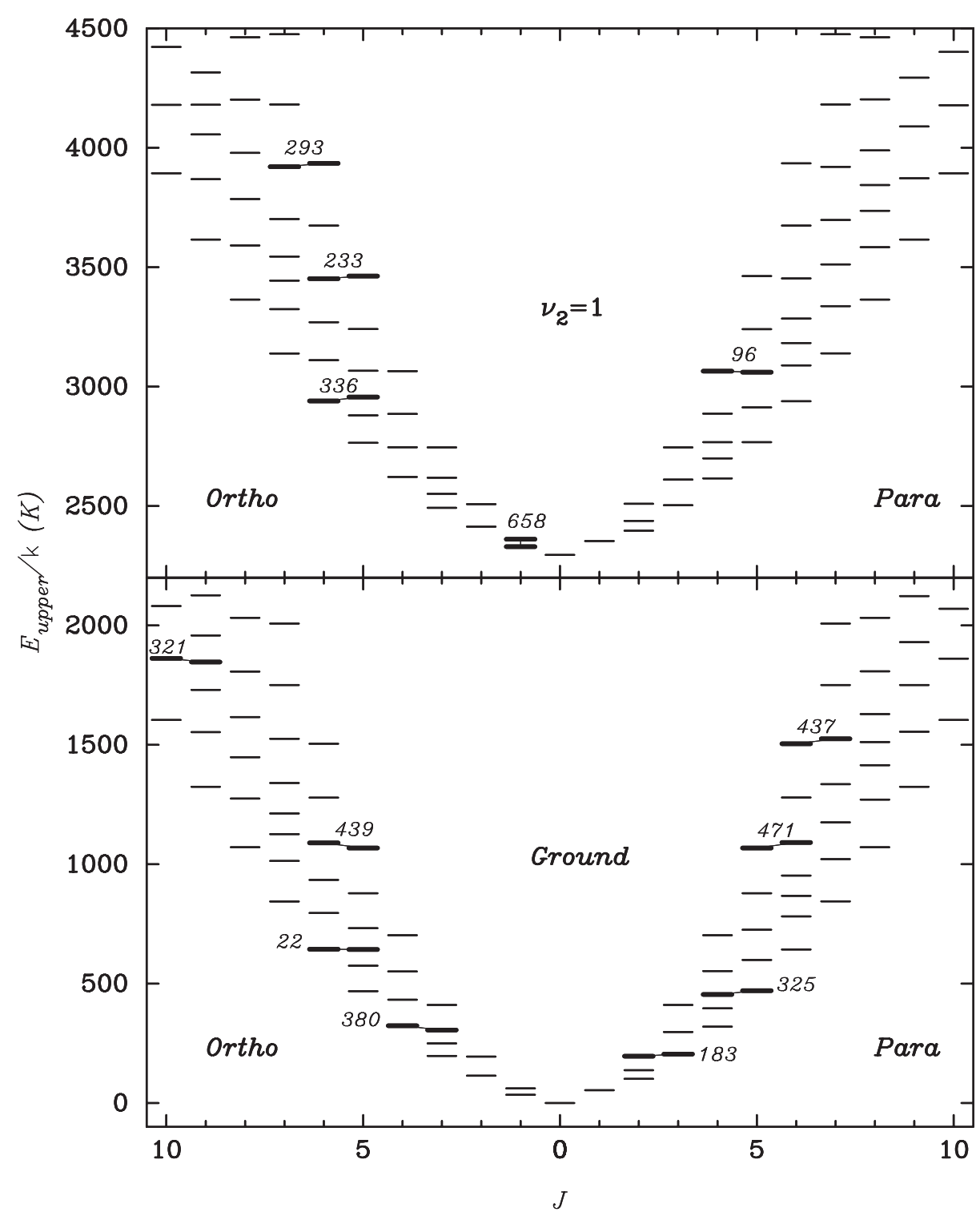

Figure 1. $\mathrm{H}_{2} \mathrm{O}$ Energy Level Diagram. Rotational levels in the ground and vibrationally-excited $\nu_{2}$ states are shown for energies between 0 to $2200 \mathrm{~K}$, and 2200 to $4500 \mathrm{~K}$ respectively. Levels of maser transitions are plotted in bold, and labels are the transition frequencies in gigahertz. The maser at $355 \mathrm{GHz}$, at an energy of $5782 \mathrm{~K}$ above ground-state, is not shown on this plot. Ortho- $\mathrm{H}_{2} \mathrm{O}$ plotted for values of the total molecular angular momentum $J$ increasing to the left, para- $\mathrm{H}_{2} \mathrm{O}$ to the right. Data are from the experimentally-derived energy levels of Tennyson et al. (2001), available on http://www.tampa.phys.ucl.ac.uk/ftp/astrodata/water/levels.

closer to the terminal velocity of the envelope (tangential vs. radial amplification as the envelope becomes denser at greater radii). Masers at 437, 439 and $471 \mathrm{GHz}$ have all been detected in CSEs and the $437 \mathrm{GHz}$ line has been found exclusively in this environment (Melnick et al. 1993; YFG97). Masers from the $\nu_{2}=1$ state, at 96, 233, 293, (336) \& 


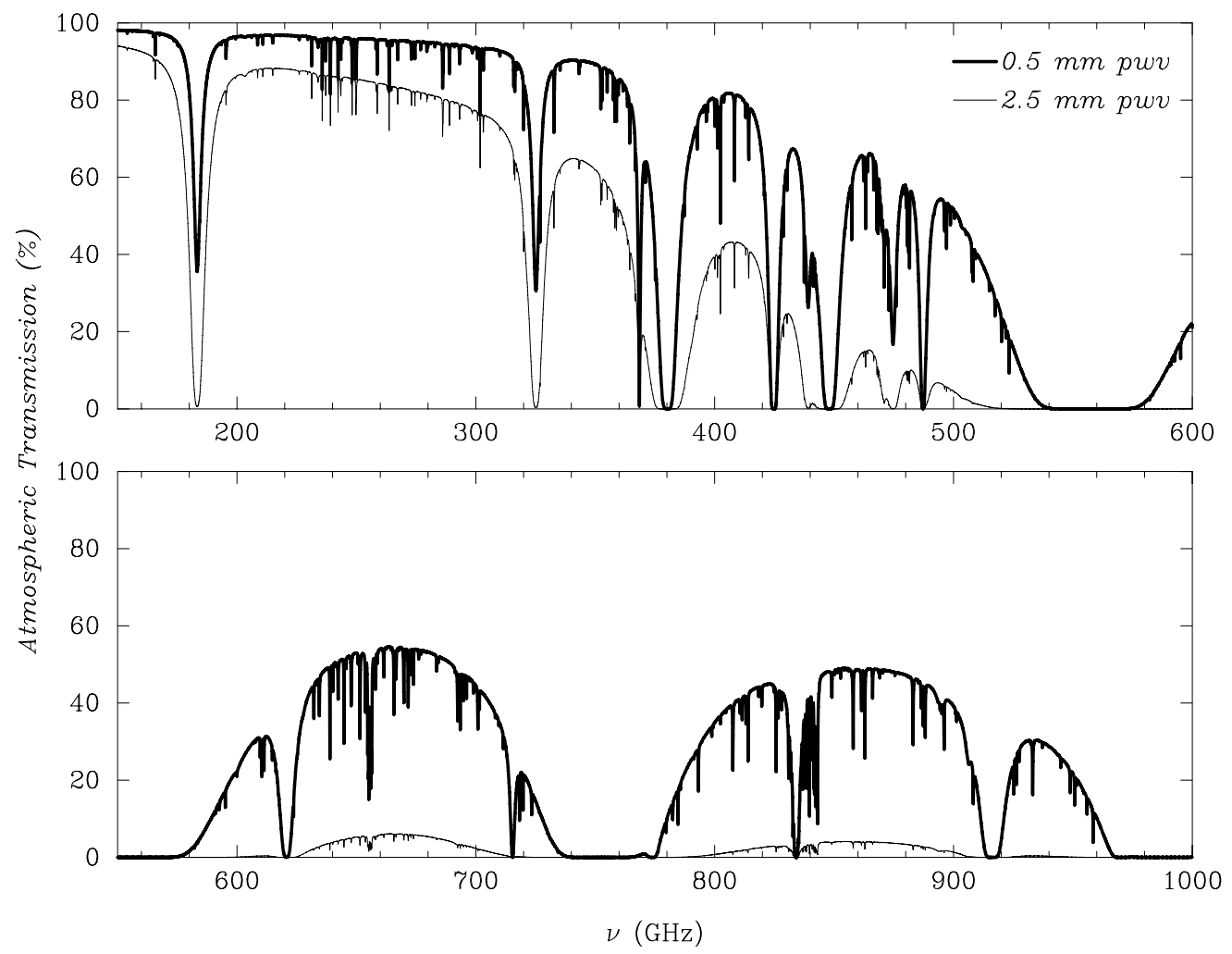

Figure 2. Zenith atmospheric transmission at Mauna Kea for column densities of 0.5 and $2.5 \mathrm{~mm} \mathrm{H}_{2} \mathrm{O}\left(\tau_{225 G H z} \sim 0.04\right.$ and 0.13 respectively). Data are from the Caltech Submillimeter Observatory Atmospheric Transmission Interactive Plotter (http://www.submm.caltech.edu/ cso/weather/atplot.shtml).

$658 \mathrm{GHz}$, are only known to occur strongly from CSEs, although $658 \mathrm{GHz}$ emission of undetermined nature is observed toward Orion-KL (Schilke et al. 2001). On the basis of excitation arguments, and similarity with $\mathrm{SiO}$ maser lineshapes in some cases, the $\nu_{2}=1$ masers likely occur close (within a few $\mathrm{R}_{*}$ ) to the central star. In recent Atacama Pathfinder Experiment (APEX) observations towards VY CMa, Menten et al. (2006) find weak maser emission from the $293 \mathrm{GHz}$ line, a non-detection of emission at $297 \mathrm{GHz}$ and thermal emission at $336 \mathrm{GHz}$. For a discussion of SMA observations of the $658 \mathrm{GHz}$ masers, often particularly strong e.g., 3000 Jy toward VY CMa, see the review by Hunter in these proceedings.

\section{2. $\mathrm{H}_{2} \mathrm{O}$ masers in star-forming regions}

Observations of the (sub)millimeter $\mathrm{H}_{2} \mathrm{O}$ masers are summarized in Table 2. 183, 321, 325, 439 and $471 \mathrm{GHz}$ masers have been observed towards high-mass star-forming regions, the 183 and $325 \mathrm{GHz}$ lines have also been observed towards low-mass star-forming regions (HH7-11A, L1448IRS3, L1448-mm at $183 \mathrm{GHz}$; IRAS16293-2422 at $325 \mathrm{GHz}$ ). The velocity range covered by the $321 \mathrm{GHz}$ maser is typically smaller than that observed at 22,183 , and $325 \mathrm{GHz}$. The $321 \mathrm{GHz}$ emission is typically weakest of these four lines, and the $22 \mathrm{GHz}$ is the strongest.

First arcsecond resolution observations of $\mathrm{H}_{2} \mathrm{O}$ masers towards a star-forming region were performed at $325 \mathrm{GHz}$ towards Orion-KL by Greenhill et al. (2007) using the compact configuration of the SMA, and followed up with a higher resolution (0.'65 circular), 
Table 2. Some (Sub)millimeter $\mathrm{H}_{2} \mathrm{O}$ observations towards star-forming regions

\begin{tabular}{|c|c|c|c|}
\hline $\begin{array}{l}\text { Freq. } \\
\text { (GHz) }\end{array}$ & Sources & $\begin{array}{c}\text { Telescope }^{1} \\
\text { (Beam) }\end{array}$ & Comments \\
\hline \multirow[t]{12}{*}{183} & Orion-KL & $\mathrm{KAO}\left(7^{\prime} .5\right)$ & First $183 \mathrm{GHz}$ detection (Waters et al. 1980 ) \\
\hline & Orion-KL, Cep A, & IRAM & Established $183 \mathrm{GHz}$ maser emission widespread \\
\hline & W49N, S252A, S158, & $30-\mathrm{m}$ & (Cernicharo et al. 1990$)$ \\
\hline & $\begin{array}{l}\text { HH7-11A, W3(H2O) } \\
\text { NGC 7538S, RN013 }\end{array}$ & & \\
\hline & Orion & IRAM & Spatially-extended emission; strong, narrow \\
\hline & & $30-\mathrm{m}$ & features at IRC2 (Cernicharo et al. 1994) \\
\hline & W49N & IRAM & Spatially-extended; less time-variable than at \\
\hline & & $30-\mathrm{m}$ & 22 \& $325 \mathrm{GHz}$ (González-Alfonso et al. 1995) \\
\hline & HH7-11 & IRAM & $183 \mathrm{GHz}$ maser variability in low-mass star \\
\hline & L1448-mm & $30-\mathrm{m}$ & formation Cernicharo et al. (1996) \\
\hline & Sgr B2 & IRAM & Strong toward cores; moderate emission at Sgr \\
\hline & & $30-\mathrm{m}$ & B2 main condensations (Cernicharo et al. 2006a) \\
\hline \multirow[t]{4}{*}{321} & $\mathrm{~W} 3(\mathrm{OH}), \mathrm{W} 49 \mathrm{~N}$, & $\mathrm{CSO}$ & Strongest $22 \mathrm{GHz} \& 321 \mathrm{GHz}$ features generally \\
\hline & W51 IRS2 \& Main & $\left(23^{\prime \prime}\right)$ & at similar velocities (Menten et al. 1990a) \\
\hline & Cep A & SMA & $22 \& 321 \mathrm{GHz}$ distributions perpendicular $(\mathrm{cm}$ \\
\hline & & $(0.75)$ & \& submm obs. $\sim 1 \mathrm{mth}$ apart) (Patel et al. 2007) \\
\hline \multirow[t]{9}{*}{325} & Orion-KL & $\mathrm{CSO}$ & $22 \& 325 \mathrm{GHz}$ cover similar velocity extents \\
\hline & W49N, W51 Main & $\left(22^{\prime \prime}\right)$ & (Menten et al. 1990b) \\
\hline & IRAS $16293-2422$ & & \\
\hline & G34.3-0.2, W49N, & $\mathrm{CSO}$ & $325,439 \& 470 \mathrm{GHz}$ cover similar velocity extents \\
\hline & Sgr B2 & & (Melnick et al. 1993) \\
\hline & Orion-KL & $\mathrm{CSO}$ & $\begin{array}{l}325 \mathrm{GHz} \text { emission much less extended than at } \\
183 \mathrm{GHz} \text { (Cernicharo et al. } 1999 \text { ) }\end{array}$ \\
\hline & Orion-KL & SMA & In high-mass protostar Source I outflow, $325 \mathrm{GHz}$ \\
\hline & & $(0 . \prime 65)$ & emission more collimated than $22 \mathrm{GHz}(\mathrm{cm} \mathrm{\&}$ \\
\hline & & Full Stokes & submm obs. $\sim 5$ yrs apart) (Greenhill et al. 2007) \\
\hline \multirow{2}{*}{$\begin{array}{l}439, \\
471\end{array}$} & G34.3-0.2 & $\mathrm{CSO}$ & First detections: $325,439 \& 470 \mathrm{GHz}$ cover \\
\hline & W49N, Sgr B2 & $\left(16^{\prime \prime}\right)$ & similar velocity extents (Melnick et al. 1993) \\
\hline
\end{tabular}

${ }^{1} \mathrm{KAO}=$ Kuiper Airborne Observatory; IRAM = Institut de Radioastronomie Millimétrique; $\mathrm{CSO}=$ Caltech Submillimeter Observatory; SMA = Submillimeter Array

full polarization epoch. In previous mapping of this region using the Caltech Submillimeter Observatory (CSO) with a $22^{\prime \prime}$ beam, Cernicharo et al. (1999) concluded that the $325 \mathrm{GHz}$ emission traces extended, low-density material of $\mathrm{n}\left(\mathrm{H}_{2}\right) \sim 10^{5-6} \mathrm{~cm}^{-3}$. However, Greenhill et al. (2007) find that it also arises from compact high-density clumps, much as the $22 \mathrm{GHz}$ transition, although in the outflow of high-mass protostar Source I the $325 \mathrm{GHz}$ emission appears more collimated. Line ratios of these $\mathrm{H}_{2} \mathrm{O}$ transitions could therefore be valuable diagnostics for shocked material in protostellar outflows.

Using the SMA, Patel et al. (2007) imaged $321 \mathrm{GHz} \mathrm{H}_{2} \mathrm{O}$ maser emission towards high-mass star-forming region Cepheus A with a resolution of 0. " 75 , in close time proximity to Very Large Array observations of the $22 \mathrm{GHz} \mathrm{H}_{2} \mathrm{O}$ masers (43 days later). The majority of $321 \mathrm{GHz}$ maser spots did not appear to be associated with those at $22 \mathrm{GHz}$, and the position angles of the roughly linear structures traced by the masers appeared perpendicular, perhaps tracing a jet and disk respectively. Patel et al. (2007) interpret the submillimeter masers in Cepheus A to be tracing significantly hotter regions (600$2000 \mathrm{~K}$ ) than the centimeter masers, see the contribution by Patel in these proceedings for further details. 


\subsection{Extragalactic $\mathrm{H}_{2} \mathrm{O}$ masers}

There have been two recent detections of extragalactic $\mathrm{H}_{2} \mathrm{O}$ masers at $183 \mathrm{GHz}$. Humphreys et al. (2005) detected emission toward the well-known $22 \mathrm{GHz} \mathrm{H}_{2} \mathrm{O}$ megamaser galaxy NGC 3079 using the SMA. At a distance of 14 Mpc, NGC 3079 harbors an active galactic nucleus (AGN), and additionally has some starburst indicators. Spatially and kinematically the $183 \mathrm{GHz}$ emission is associated with the AGN, with emission peaking at the same position as that of $22 \mathrm{GHz}$ emission imaged by Kondratko et al. (2005) using VLBI. At $22 \mathrm{GHz}$, the emission has a time-variable peak flux density in the range 3-12 Jy, whereas at $183 \mathrm{GHz}$, the $\mathrm{H}_{2} \mathrm{O}$ maser emission had a peak flux density of $\sim 0.5 \mathrm{Jy}$. Humphreys et al. (2005) also make a tentative detection of the $439 \mathrm{GHz}$ maser using the JCMT.

Cernicharo, Pardo \& Weiss (2006) detected a megamaser at $183.310 \mathrm{GHz}$ in Arp 220 using the IRAM $30 \mathrm{~m}$, with a line width of $\sim 350 \mathrm{~km} \mathrm{~s}^{-1}$ and total luminosity of $\sim 2.5 \times$ $10^{8} \mathrm{~K} \mathrm{~km} \mathrm{~s}^{-1} \mathrm{pc}^{2}$. This is very interesting since no emission at $22 \mathrm{GHz}$ has been detected from Arp 220 (an $\mathrm{OH}$ megamaser source). This fact puts constraints on the physical conditions of the central region of Arp 220, which are further strengthened by observations of HCN and HNC $J=3-2$ and $J=1-0$, suggesting densities of $\mathrm{n}\left(\mathrm{H}_{2}\right)=10^{5}$ $\mathrm{cm}^{-3}$. Cernicharo, Pardo \& Weiss (2006) propose a scenario with $\sim 10^{6}$ star-forming cores similar to those found in Sgr B2 in the central kiloparsec of Arp 220. The $183 \mathrm{GHz}$ line is therefore an additional tool to explore the physical conditions in starburst and AGN sources, with the potential for high angular resolution observations using ALMA.

\section{3. (Sub)millimeter $\mathrm{SiO}$ masers in evolved stars}

(Sub)millimeter ${ }^{28} \mathrm{SiO}$ masers have been detected from the $J=5-4 \approx 215 \mathrm{GHz}(v=1$ $\& 2$, Clemens \& Lane 1983; $v=3$ tentative detection from VX Sgr, Jewell et al. 1987; $v=$ 3 \& 4 from VY CMa, Cernicharo, Bujarrabal \& Santaren 1993), $J=6-5 \approx 258 \mathrm{GHz}$ $(v=1$, Jewell et al. 1987; $v=2$, VY CMa, Cernicharo, Bujarrabal \& Santaren 1993), $J=7-6 \approx 301 \mathrm{GHz}(v=1 \& 2, \mathrm{R}$ Aqr, Gray et al. 1995), $J=8-7 \approx 344 \mathrm{GHz}$ $(v=1$, VY CMa, and tentative $v=2$, Humphreys et al. 1997; $v=2$, VY CMa, Gray, Humphreys \& Yates 1999). The highly-rotationally excited masers are very rare from the $v=3 \& 4$ states (Pardo et al. 1998 and references therein) which lie at $>5400 \mathrm{~K}$ above ground state. They are more common in the $v=1 \& 2$ (Jewell et al. 1987; Cernicharo, Bujarrabal \& Santaren 1993; Humphreys et al. 1997; Gray, Humphreys \& Yates 1999) especially ing $J=5$-4 emission, but weaker than their lower frequency counterparts in the same vibrational states, and more time-variable. In a survey of 34 supergiant and longperiod variable stars, Gray, Humphreys \& Yates (1999) found that for Mira variables, emission from the high-frequency transitions is absent or weak from optical phase range $\phi \sim 0.4-0.7$ of the stellar pulsation cycle.

$\mathrm{SiO}$ maser emission at lower frequencies is well-known to display high degrees (tens of $\%$ ) of linear polarisation e.g., $v=1 J=1-0(43 \mathrm{GHz})$ maser components can be $\sim 100 \%$ linearly polarized (e.g., Kemball \& Diamond 1997). Using a partially-completed SMA, Shinnaga et al. (2004) imaged the $v=1, J=5-4 \mathrm{SiO}$ maser emission of supergiant VY $\mathrm{CMa}$ to investigate linear polarization properties at higher frequency. The majority of components showed significant degrees of linear polarization, with one at the $60 \%$ level, that Shinnaga et al. attribute to a radiative pumping process.

For the less abundant isotopomers ${ }^{29} \mathrm{SiO}$ and ${ }^{30} \mathrm{SiO}$, Cernicharo \& Bujarrabal (1992) detected maser emission from the $v=0 J=5-4$ transition for both species, the ${ }^{29} \mathrm{SiO}$ $v=2, J=6-5$ line, and the ${ }^{30} \mathrm{SiO} v=1 J=6-5$ towards VY CMa. For ${ }^{29} \mathrm{SiO}$, 
the $v=3 J=8-7$ at $335.9 \mathrm{GHz}$ was detected toward TX Cam, R Leo and W Hya at optical stellar phases $\phi$ of 0.3, 0.15 and 0.25 respectively (González-Alfonso et al. 1996) and towards VY CMa (González-Alfonso et al. 1996; Menten et al. 2006 using APEX). Menten et al. (2006) also detected maser emission in the ${ }^{30} \mathrm{SiO} v=1 \mathrm{~J}=8-7$ line towards VY CMa, whereas the ${ }^{29} \mathrm{SiO} v=0 J=8-7$ transition appears thermal. Infrared line overlaps of the $\mathrm{SiO}$ isotopomers is believed to be important in the pump scheme of these masers (e.g., Herpin \& Baudry 2000). For a detailed discussion of $\mathrm{SiO}$ masers in evolved stars, see the review by Bujarrabal in these proceedings.

\section{4. (Sub)millimeter $\mathbf{H}$ recombination masers}

Hydrogen recombination maser emission is known from two galactic peculiar stellar sources, MWC 349A (Martin-Pintado et al. 1989) and Eta Carinae (Cox et al. 1995). (Sub)-millimeter maser emission from MWC 349A has been detected from at least the $\mathrm{H} 31 \alpha(210.5 \mathrm{GHz}), \mathrm{H} 30 \alpha(231.9 \mathrm{GHz}), \mathrm{H} 29 \alpha(256.302 \mathrm{GHz})$ (Martin-Pintado et al. 1989) from H26 $\alpha$ (353.623 GHz; Thum et al. 1994a) and the H21 $\alpha$ (662.405 GHz; $350 \mathrm{Jy}$; Thum et al. 1994b), H32 $\beta$ (366.6 GHz; Thum et al. 1995). Planesas, Martin-Pintado \& Serabyn (1992) spatially resolved the double-peaked maser spectrum into two emitting regions, separated by 0 !'065, associated with the red and blue-shifted emission from a sub-arcsecond disk imaged in the near-infrared by Danchi, Tuthill \& Monnier (2001). Weintroub et al. (2007) again detected $\mathrm{H} 30 \alpha$ and $\mathrm{H} 26 \alpha$ maser emission from the two regions using the SMA, but also found emission at positions between them with an accuracy of $0{ }^{\prime \prime} 01$. The emission position-velocity diagram is consistent with that of an edge-on disk in approximate Keplerian rotation. However, Weintroub et al. (2007) argue that systematic deviation from Keplerian rotation may indicate the presence of spiral structure in the MWC 349A disk (see also these proceedings). From Zeeman observations of the H30 $\alpha$ maser, Thum \& Morris (1999) report a dynamically-important magnetic field associated with the corona of the circumstellar disk, possibly generated by a local disk dynamo. Pumping of the masers in MWC 349A has been explained by Strelnitski et al. (1996). Towards Eta Carinae, Cox et al. (1995) detected millimeter maser emission at $\mathrm{H} 30 \alpha, \mathrm{H} 29 \alpha$ and $\mathrm{H} 37 \beta(240.021 \mathrm{GHz}$ ) (see also Abraham et al. (2002)).

Extragalactic $\mathrm{H}$ recombination maser emission from the $\mathrm{H} 27 \alpha(316.416 \mathrm{GHz})$ transition has also been detected towards M82 (Seaquist et al. 1996). The emission is highly time-variable, and of peak flux density $1.5 \mathrm{Jy}$ at the strongest epoch. We note that $\mathrm{H}$ recombination masers at lower frequency may also have been detected from starburst galaxies, see references in Seaquist et al. (1996), and that $\mathrm{H}$ recombination masers are predicted to probe the Epochs of Recombination and Reionisation (Spaans \& Norman 1997).

\section{5. (Sub)millimeter $\mathrm{CH}_{3} \mathrm{OH}$ masers}

In a survey of Galactic star-forming regions, Kalenskii, Slysh \& Val'Tts (2002) detected maser emission from methanol $8_{-1}-7_{0}$ E at $229.8 \mathrm{GHz}$ towards DR $21(\mathrm{OH})$ and DR 21 West, and toward two maser candidates, L 379IRS3 and NGC 6334I(N). The maser emission in DR21(OH) and DR 21 West indicates gas kinetic temperatures of $\mathrm{T}_{k} \sim 50 \mathrm{~K}$ and densities of $\mathrm{n}\left(\mathrm{H}_{2}\right)=3 \times 10^{4} \mathrm{~cm}^{-3}$. Towards 16 other sources, the emission detected from this line was thermal in nature. Sobolev et al. (2002) reported the detection of class II methanol emission at $216.9 \mathrm{GHz}$, and models by Cragg et al. (2005) predict the existence of many more (sub)millimeter Class II methanol masers. 


\section{6. (Sub)millimeter HCN \& SiS masers in carbon stars}

(Sub)millimeter HCN maser emission has been detected from carbon-rich circumstellar envelopes. Using the Caltech Submillimeter Observatory (CSO), Schilke, Mehringer \& Menten (2000) and Schilke \& Menten (2003) detected the $J=9-8$ maser of the $\left(04^{0} 0\right)$ vibrationally-excited state of $\mathrm{HCN}$ at a frequency of $\approx 804.751 \mathrm{GHz}$ towards IRC +10216 (at two epochs of peak flux densities 1420 \& $840 \mathrm{Jy}$ ) and CIT 6 (110 Jy). The lower level of the maser is at $4200 \mathrm{~K}$ above ground state, such that emission should originate from the innermost region of the CSEs $\left(<3.5 \mathrm{R}_{*}\right)$. Schilke \& Menten $(2003)$ also detected the $\left(11^{1} 0\right)-\left(04^{0} 0\right), J=10-9$ maser at $890.761 \mathrm{GHz}$ towards IRC+10216 (at four epochs with peak flux densities of 6120, 4430, 9230, 900 Jy), CIT 6 (1090 \& 1150 Jy) and Y CVn (140 Jy). In surveys using the Heinrich-Hertz-Submillimeter Telescope, Bieging, Shaked \& Gensheimer (2000) and Bieging (2001) discovered maser emission in the $J=3-2$ $(265.886 \mathrm{GHz})$ and $4-3(354.505 \mathrm{GHz})$ transitions of the $\mathrm{HCN}\left(01^{1 c} 0\right)$ vibrational bending mode toward five stars: R Scl, V384 Per, R Lep, Y CVn, and V Cyg (out of 12 observed). Submillimeter HCN masers at 964 and $968 \mathrm{GHz}$ are also predicted by Schilke \& Menten (2003), and could be detected using SOFIA.

SiS masers were first discovered by Henkel, Matthews \& Morris (1983) from the $v=0$, $J=1-0$ transition at $18 \mathrm{GHz}$ toward carbon-rich star IRC +10216 . (Sub)millimeter SiS maser emission was also detected toward IRC+10216 from the $v=0, J=11-10$ $(199.672 \mathrm{GHz}), J=14-13(254.103 \mathrm{GHz})$ and $J=15-14(272.243 \mathrm{GHz})$ transitions by Fonfría Expósito et al. (2006) using the IRAM 30-m. Line overlap is believed to be important in the pumping scheme of the highly-rotationally excited masers and they are thought to occupy $\sim 5-7 \mathrm{R}_{*}$ in the CSE of IRC+10216. Future high-resolution observations of the HCN and SiS masers using ALMA will therefore yield new information on the dust formation zone of carbon stars.

\section{Summary \& future propects}

Observations of submillimeter masers at high angular resolution provide new means of studying stellar evolution, star formation and AGN/starburst activity. Where different maser transitions trace the same gas, we will be able to place new constraints on radiative transfer models to determine small-scale source temperature and density distributions. Where maser lines trace different regions of sources, we will be able to map out more of source structures and dynamics than ever before. Submillimeter masers could be particularly important probes of regions in which longer wavelength maser emission is subject to obscuration e.g., due to free-free or synchrotron opacity.

The spatial resolution and sensitivity of ALMA will revolutionize submillimeter science. There have also been huge strides in submillimeter VLBI, with fringes obtained at 129, 147, and $230 \mathrm{GHz}$ (see e.g., Krichbaum et al. (2007)) and with imaging of $\mathrm{SiO}$ $J=3-2$ masers at $129 \mathrm{GHz}$ in VY CMa and several AGB stars already achieved (Doeleman et al. 2005; Doeleman, private communication). Within the next decade, observations of submillimeter masers are likely to become very much more commonplace and, in conjunction with detailed modelling, will yield a wealth of new and exciting avenues of research.

\section{Acknowledgements}

EH thanks Lincoln Greenhill, Preethi Pratap, Andrej Sobolev, Vladimir Strelnitski and Jonathan Weintroub for providing unpublished results, and Jim Moran for helpful comments on this manuscript. 


\section{References}

Abraham, Z., Damineli, A., Durouchoux, P., Nyman, L., \& McAuliffe, F. 2002, in: V. Migenes \& M. J. Reid (eds.), Cosmic Masers: From Proto-Stars to Black Holes, IAU Symposium, vol. 206 , p. 234

Alcolea, J. \& Menten, K. M. 1993, in: A. W. Clegg \& G. E. Nedoluha (eds.), Astrophysical Masers, Lecture Notes in Physics (Berlin: Springer Verlag), vol. 412, p. 399

Bieging, J. H., Shaked, S., \& Gensheimer, P. D. 2000, ApJ, 543, 897

Bieging, J. H. 2001, ApJ, 549, L125

Cernicharo, J., Thum, C., Hein, H., John, D., Garcia, P., \& Mattioco, F. 1990, A\&\&A, 231, L15

Cernicharo, J. \& Bujarrabal, V. 1992, ApJ, 401, L109

Cernicharo, J., Bujarrabal, V., Santaren, J. L. 1993, ApJ 407, L33

Cernicharo, J., González-Alfonso, E., Alcolea, J., Bachiller, R., John, D. 1994, ApJ, 432, L59

Cernicharo, J., Bachiller, R., González-Alfonso, E. 1996, A\&3A, 305, L5

Cernicharo, J., Pardo, J. R., González-Alfonso, E., Serabyn, E., Phillips, T. G., Benford, D. J., Mehringer, D. 1999, ApJ, 520, L131

Cernicharo, J., Goicoechea, J. R., Pardo, J. R., Asensio-Ramos, A. 2006a, ApJ, 642, 940

Cernicharo, J., Pardo, J. R., \& Weiss, A. 2006b, ApJ, 646, L49

Cheung, A. C., Rank, D. M., Townes, C. H., Thornton, D. D., Welch, W. J. 1969, Nature, 221, 626

Clemens, D. P. \& Lane, A. P. 1983, ApJ, 266, L117

Cooke, B. \& Elitzur, M. 1985, ApJ, 295, 175

Cox, P., Martin-Pintado, J., Bachiller, R., Bronfman, L., Cernicharo, J., Nyman, L.-A., Roelfsema, P. R. 1995, A\& A, 295, L39

Cragg, D. M., Sobolev, A. M., Godfrey, P. D. 2005, MNRAS, 360, 533

Danchi, W. C., Tuthill, P. G., \& Monnier, J. D. 2001, ApJ, 562, 440

Deguchi, S. 1977, PASJ, 29, 669

Deguchi, S., Nguyen-Q-Rieu 1990, ApJ, 360, L27

Doeleman, S. S., Phillips, R. B., Rogers, A. E. E., et al. 2005, in: J. Romney \& M. Reid (eds.), Future Directions in High Resolution Astronomy, Astronomical Society of the Pacific Conference Series, vol. 340, p. 605

Feldman, P. A., Matthews, H. E., Cunningham, C. T., Hayward, R. H., Wade, J. D., Amano, T., Scappini, F. 1991, JRASC, 85, 191

Feldman, P. A., Matthews, H. E., Amano, T., Scappini, F., Lees, R. M. 1993, in: A. W. Clegg \& G. E. Nedoluha (eds.), Astrophysical Masers, Lecture Notes in Physics (Berlin: Springer Verlag), vol. 412 , p. 65

Fonfría Expósito, J. P., Agundez, M., Tercero, B., Pardo, J. R., Cernicharo, J. 2006, ApJ, 646, L127

González-Alfonso, E., Cernicharo, J., Bachiller, R., Fuente, A. 1995, A\&\&A, 293, L9

González-Alfonso, E., Alcolea, J., Cernicharo, J. 1996, A\& A, 313, L13

González-Alfonso, E., Cernicharo, J., Alcolea, J., \& Orlandi, M. A. 1998, A\&̛A, 334, 1016

Gray, M. D., Ivison, R. J., Yates, J. A., Humphreys, E. M. L., Hall, P. J., \& Field, D. 1995, MNRAS, 277, L67

Gray, M. D., Humphreys, E. M. L., \& Yates, J. A 1999, MNRAS, 304, 906

Greenhill, L. J., et al. 2007, in prep

Henkel, C., Matthews, H. E., \& Morris, M. 1983, ApJ, 267, 184

Herpin, F. \& Baudry, A. 2000, A\&A, 359, 1117

Humphreys, E. M. L., Gray, M. D., Yates, J. A., Field, D. 1997, MNRAS, 287, 663

Humphreys, E. M. L., Yates, J. A., Gray, M. D., Field, D., Bowen, G. H. 2001, A\&\&A, 379, 501

Humphreys, E. M. L., Greenhill, L. J., Reid, M. J., Beuther, H., Moran, J. M., Gurwell, M., Wilner, D. J., Kondratko, P. T. 2005, ApJ, 634, L133

Ivison, R. J., Yates, J. A., Hall, P. J. 1998, MNRAS, 295, 813

Jewell, P. R. and Dickinson, D. F. and Snyder, L. E. and Clemens, D. P. 1987, ApJ, 323, 749

Kalenskii, S. V., Slysh, V. I., Val'tts, I. E. 2002, ARep, 46, 49

Kemball, A. J., \& Diamond, P. J. 1997, ApJ, 481, L111

Kondratko, P. T., Greenhill, L. J., \& Moran, J. M. 2005, ApJ, 618, 618 
Krichbaum, T. P., Graham, D. A., Witzel, A., et al. 2007, in: Towards the Event Horizon: High Resolution VLBI Imaging of Nuclei of Active Galaxies, Exploring the Cosmic Frontier, ESO Astrophysics Symposia European Southern Observatory, Springer, p. 189

Martin-Pintado, J., Bachiller, R., Thum, C., Walmsley, M. 1989, 215, L13

Menten, K. M. \& Melnick, G. J. 1989, ApJ, 341, L91

Melnick, G. J., Menten, K. M., Phillips, T. G., Hunter, T. 1993, ApJ, 416, L37

Menten, K. M., Melnick, G. J., Phillips, T. G. 1990b, ApJ, 350, L41

Menten, K. M., Melnick, G. J., Phillips, T. G., Neufeld, D. A. 1990a, ApJ, 363, L27

Menten, K. M., \& Young, K. 1995, ApJ, 450, L67

Menten, K. M., Philipp, S. D., Güsten, R., Alcolea, J., Polehampton, E. T., Brünken, S. 2006, $A \mathscr{E} A, 454, \mathrm{~L} 107$

Neufeld, D. A., Melnick, G. J. 1991, ApJ, 368, 215

Pardo, J. R., Cernicharo, J., Gonzalez-Alfonso, E. and Bujarrabal, V. 1998, A\&A, 329, 219

Patel, N. A., Curiel, S., Zhang, Q., Sridharan, T. K., Ho, P. T. P., \& Torrelles, J. M. 2007, ApJ, $658, \mathrm{~L} 55$

Phillips, T. G., Kwan, J., Huggins, P. J. 1980, in: B. H. Andrew (eds.), Interstellar Molecules, IAU Symposium, vol. 87, p. 21

Planesas, P., Martin-Pintado, J., Serabyn, E., 1992, ApJ, 386, L23

Schilke, P., Mehringer, D. M., \& Menten, K. M. 2000, ApJ, 528, L37

Schilke, P., Benford, D. J., Hunter, T. R., Lis, D. C., Phillips, T. G. 2001, ApJS, 132, 281

Schilke, P., \& Menten, K. M. 2003, ApJ, 583, 446

Seaquist, E. R., Carlstrom, J. E., Bryant, P. M., Bell, M. B. 1996, ApJ, 465, 691

Shinnaga, H., Moran, J. M., Young, K. H., Ho, P. T. P. 2004, ApJ, 616, L47

Sobolev, A. M., Ostrovskii, A. B., Malyshev, A. V., Cragg, D. M., Godfrey, P. D., Sutton, E. C., Watson, W. D., Ellingsen, S. P., Caswell, J. L. 2002, in: V. Migenes \& M. J. Reid (eds.), Cosmic Masers: From Proto-Stars to Black Holes, IAU Symposium, vol. 206, p. 179

Spaans, M., \& Norman, C. A. 1997, ApJ, 488, 27

Strelnitski, V. S., Ponomarev, V. O., Smith, H. A. 1996, ApJ, 470, 1118

Tennyson, J., Zobov, N. F., Williamson, R., Polyansky, O.L., Bernath, P. F. 2001, J. Phys. Chem. Ref. Data, 30, 735

Thum, C., Matthews, H. E., \& Martin-Pintado, J., Serabyn, E., Planesas, P., Bachiller, R. 1994a, $A \mathscr{E} A, 283,582$

Thum, C., Matthews, H. E., Harris, A. I., Tacconi, L. J., Schuster, K. F., Martin-Pintado, J. 1994b, A\&A, 288, L25

Thum, C., Strelnitski, V. S., Martin-Pintado, J., Matthews, H. E., Smith, H. A. 1995, A\&A, 300,843

Thum, C. \& Morris, D. 1999, A\&A, 344, 923

Waters, J. W., Kakar, R. K., Kuiper, T. B. H., Roscoe, H. K., Swanson, P. N., Rodriguez Kuiper, E. N., Kerr, A. R., Thaddeus, P., Gustincic, J. J. 1980, ApJ, 235, 57

Weintroub, J., et al. 2007, in prep

Yates, J. A., Cohen, R. J., Hills, R. E., 1995, MNRAS, 273, 529

Yates, J. A. \& Cohen, R. J. 1996, MNRAS, 278, 655

Yates, J.A., Field, D., \& Gray, M.D. 1997, MNRAS, 285, 303 